\title{
Effects of reinforcement shifts upon subsequent saccharin consumption'
}

\author{
RONALD GANDELMAN and JAY A. TROWILL. Universit! \\ of Massachusetts, Amherst, Mass. $0100 ?$
}

An elation effect in terms of home-cage saccharin drinking was seen when nondeprived rats were shifted from saccharin to water and back to saccharin.

Durable elation and depression effects (overtesponding when shifted from a low to high reward and underresponding when shifted from a high to low reward, respectively) have recently been found by Panksepp \& Trowill (in press) when shifting current intensities of rewarding electrical brain stimulation. These results are interesting since elation effects have been rarely reported when deprived animals are reinforced with deprivation-specific rewards (Bolles, 1967 ). The authors concluded that deprivation conditions may elevate response rates to a level which precludes further elevation of responding contingent upon increasing the reward (i.e., elation). This problem was eliminated by employing nondeprived animals responding for brain stimulation. If this interpretaion is valid. elation should be scen with sacharin drinking, a response which also can be maintained without deprivation.

\section{SUBJECTS}

Seven male Charles River albino rats were individually housed and maintained on ad lib food (Purina Lab Chow pellets) and water throughout the experiment. All tests were run in the homecages.

\section{PRETRAINING}

A bottle containing .25\% (w/v) saccharin solution (sodium saccharin and plain tap water) was attached to each cage for I h a day for six days. All Ss began to drink by the second day.

\section{TESTING}

For 13 days a bottle containing a measured amount of the saccharin solution was attached to the cage for $1 \mathrm{~h}$. This was followed by $1 \cdot h$ presentations of plain tap water for three days. Finally, seven more days of saccharin were given ( $1 \mathrm{~h}$ per day). The amount of intake was recorded for each session.

\section{RESULTS}

Figure 1 shows the day-by-day mean pre- and postwater saccharin intakes as well as the mean water intakes. As can be seen, postwater saccharin consumption is generally gredter than the prewater intakes. This difference, evaluated by the randomization test for matched pairs (Siegel, 1956), is statistically significant $(p<.02)$. For every $S$, the mean



Fig. 1. Mean saccharin and water intakes per daily session.

postwater saccharin intake was greater than the mean prewater saccharin intake.

\section{DISCUSSION}

The results show that an elation effect can be produced by interspersing a period of water presentation hetween sactharin presentations in nondeprived animals. Thus. elation can be seen when employing reinforcement which does not require deprivation conditions to maintain its relevancy. These results, in agreement with the interpretation of Panksepp \& Trowill. emphasize the need for a reevaluation of assumptions based primarily upon the behavior of deprived organisms. Furthermore, these data and those of Panksepp and Trowill show similarities between reinforcing brain stimulation and conventional rewards, a topic which is taken up in detail elsewhere (Trowill, Panksepp.\& Gandelman, in press).

\section{REFERENCES}

BOLLFS R. C. Theory of motivation. New York: Harper \& Row 1967 PANKSEPP, J.. \& TROWILL, J. A. Positive and negative contrast effects with electrical stimulation of the brain. Phy siological Behavior. in press.

SIEGEL. S. Nonparametric statistics. New York: Mc(iraw-Hill, 1950

TROWILL. J. A., PANKSEPP, J., \& GANDELMAN. R. An incentive model of rewarding brain stimulation. Psychological Review, in press. NOTE

1. This study was partially supported by U.S.P.H.S. Grant MH 13155-02 awarded to Jay $A$. Trowill. 